\title{
Prebiotic Effect of Berberine and Curcumin Is Associated with the Improvement of Obesity in Mice
}

\author{
Audrey M. Neyrinck ${ }^{1}{ }^{\circledR}$, Cándido Robles Sánchez ${ }^{1}$, Julie Rodriguez ${ }^{1}$, Patrice D. Cani ${ }^{1,2}{ }^{\circledR}$, Laure B. Bindels ${ }^{1}$ and \\ Nathalie M. Delzenne ${ }^{1, * \mathbb{D}}$ \\ 1 Metabolism and Nutrition Research Group, Louvain Drug Research Institute, Université Catholique de \\ Louvain, UCLouvain, B-1200 Brussels, Belgium; audrey.neyrinck@uclouvain.be (A.M.N.); \\ candido.robles@uclouvain.be (C.R.S.); j.rodriguez@uclouvain.be (J.R.); patrice.cani@uclouvain.be (P.D.C.); \\ laure.bindels@uclouvain.be (L.B.B.) \\ 2 Walloon Excellence in Life Sciences and BIOtechnology (WELBIO), Louvain Drug Research Institute, \\ UCLouvain, B-1200 Brussels, Belgium \\ * Correspondence: nathalie.delzenne@uclouvain.be
}

check for updates

Citation: Neyrinck, A.M.; Sánchez, C.R.; Rodriguez, J.; Cani, P.D.; Bindels, L.B.; Delzenne, N.M. Prebiotic Effect of Berberine and Curcumin Is Associated with the Improvement of Obesity in Mice. Nutrients 2021, 13, 1436. https://doi.org/ 10.3390/nu13051436

Academic Editors: Roberta Masella and Manuel Sánchez Santos

Received: 16 March 2021

Accepted: 22 April 2021

Published: 24 April 2021

Publisher's Note: MDPI stays neutral with regard to jurisdictional claims in published maps and institutional affiliations.

Copyright: (c) 2021 by the authors. Licensee MDPI, Basel, Switzerland. This article is an open access article distributed under the terms and conditions of the Creative Commons Attribution (CC BY) license (https:/ / creativecommons.org/licenses/by/ $4.0 /)$.

\begin{abstract}
Berberine and curcumin, used as food additives or food supplements, possess interesting anti-inflammatory and antioxidant properties. We tested the potential protective effect of both phytochemicals in genetically obese mice and we determined whether these effects can be related to the modulation of gut functions and microbiota. $\mathrm{Ob} / \mathrm{ob}$ mice were fed a standard diet supplemented with or without $0.1 \%$ berberine and/or $0.3 \%$ curcumin for 4 weeks. By using targeted qPCR, we found that cecal content of Bifidobacterium spp. and Akkermansia spp. increased mainly upon berberine supplementation. Genes involved in innate immunity (Pla2g2a), mucus production (Muc2) and satietogenic peptide production ( $G c g$ and $P y y$ ) were upregulated in the colon of mice treated with both phytochemicals. Berberine supplementation alone reduced food intake, body weight gain, hypertriglyceridemia and hepatic inflammatory and oxidative stress markers, thus lessening hepatic injury. The increase in Bifidobacterium spp. and Akkermansia spp. was correlated with the improvement of gut barrier function and with the improvement of hepatic inflammatory and oxidative stresses in obese mice. These data support the fact that non-carbohydrate phytochemicals may modulate the gut microbiota in obesity and related gut and hepatic alterations.
\end{abstract}

Keywords: berberine; curcumin; prebiotic; gut barrier; liver injury; obesity; inflammation

\section{Introduction}

Numerous studies in animal models but also in humans describe alterations of the gut microbial ecosystem (i.e., dysbiosis) associated with obesity and hallmarks of metabolic syndrome, including metabolic-associated fatty liver disease (MAFLD) [1-3]. The manipulation of the gut microbiota by the diet appears to be an innovative therapeutic tool to prevent or control obesity and related metabolic disorders [4]. In particular, it has been reported that bacteria such as Bifidobacterium, Lactobacillus and Akkermansia muciniphila may play a crucial role as anti-obesity bacteria in animal models and humans [1,5-8]. The impact of nutrients that change the gut microbiota composition, or that are metabolized by the gut bacteria into bioactive metabolites, is already described in different animal models of obesity. Interesting strategies to manage weight control are nutritional interventions with dietary fiber $[1,9,10]$. In particular, prebiotics are defined initially as "a non-digestible food ingredient that beneficially affects the host by selectively stimulating the growth and/or activity of one or a limited number of bacteria in the colon, and thus improves host health" [11]. Inuline-type fructans (fructo-oligosaccharides and inulin) and galactans (galacto-oligosaccharides) are considered key prebiotics through their effect on the enrichment of Lactobacillus spp. and/or Bifidobacterium spp. [12]. This definition has evolved over time, with the latest consensus being that a prebiotic is a "substrate that is 
selectively utilized by host microorganisms conferring a health benefit", thus expanding the concept to non-carbohydrate substances [12]. Phytochemicals, mostly plant polyphenols, have been proposed as potential prebiotics, but more studies are needed to elaborate how their interaction with the gut microbiota influences health effects [12]. It is estimated that 90-95\% of dietary polyphenols are not absorbed in the small intestine and, therefore, reach the colon, where they undergo significant biotransformation by the colonic microbiota, often through deglycation and hydrolysis by microbial enzymes, into metabolites that can be absorbed [13]. In fact, growing evidence indicates that the health benefits associated with polyphenol consumption depend more on their microbial metabolism to bioactive molecules than on the parent compounds themselves [13]. However, there are few data on their prebiotic effect (improvement of health with concomitant interaction with the gut microbiota) as compared to a large number of studies performed with fructo-oligosaccharides, galacto-oligosaccharides or inulin-type fructans.

Initially, polyphenols and other phytochemical compounds were extensively studied because of their strong anti-inflammatory and antioxidant properties [14]. Furthermore, preclinical and clinical studies suggest that polyphenols are able to exert antimicrobial activities against pathogenic gut bacteria [15]. Several polyphenols, such as tannins, flavonoids, phenolic acids, lignans and stilbenes, alone or in combination, are known to exhibit antibacterial activity against Gram-positive bacteria. Their mechanism of action is quite diverse, targeting biofilm formation, lipid membrane, membrane receptors, ion channels and bacterial metabolites [16]. More recently, they have attracted the interest of the research community because of their potential role in reducing obesity [17]. Interesting reviews described the effects of plant polyphenols on gut microbiota and/or obesity in preclinical studies $[14,18]$. Among these, curcumin is often studied. The dried ground rhizome of Curcuma longa is a popular dietary spice in Asia, as used in curry [19]. It is also an integral part of the Indian traditional medicine called Ayurveda. The polyphenol curcumin represents $2-8 \%$ of most curcuma preparations and is generally regarded as its most active component, having potent antioxidant, anti-inflammatory and anticarcinogenic properties [20,21]. Curcumin-based oral supplements have been proposed in the treatment of various medical inflammatory conditions, such as effluvium telogen [22]. In addition, curcumin has been shown to have protective effects on the liver against fat accumulation, mainly in animal models of high-fat (HF) diet-induced obesity [23]. However, the exact mechanisms by which curcumin reduces liver fat accumulation and alleviates hepatic steatosis are not fully understood. Curcumin also prevents HF diet-induced insulin resistance and obesity by lessening hepatic lipogenesis and inflammation in adipocytes [24]. A few studies demonstrated that dietary curcumin significantly improves obesity-associated inflammation and diabetes in a genetic model of obesity $o b / o b$ mice, but the concentration used was very high (1 to $3 \%)[25,26]$. In addition, the potential involvement of gut microbiota modulation in curcumin effects has not been demonstrated in these studies.

Berberine is another interesting prebiotic candidate. It is a natural isoquinoline-type alkaloid originally isolated from Coptis chinensis, with a long history of Chinese medicinal application [27]. Berberine suppresses proinflammatory responses by inhibiting mitogenactivated protein kinase signaling and cellular reactive oxygen species production [28]. Based on clinical and preclinical studies, findings support the hypothesis that berberine displays beneficial effects for treating obesity and suggest that the mechanism of action implies AMP-activated protein kinase [29,30].

A synergistic antimicrobial activity of berberine and curcumin has already been demonstrated against methicillin-resistant S. aureus (MRSA) [31]. In the present study, we explored the prebiotic potential of both phytochemicals, administrated alone or in combination, in a model of $o b / o b$ mice. We targeted by quantitative PCR specific bacteria such as Lactobacillus spp., Bifidobacterium spp. and Akkermansia spp., the well-known bacteria often implicated in the prebiotic effects of nutrients in the context of obesity [5-8], as well as Bacteroides spp. as a good representative dominant Gram-negative bacterium in the gut microbiota [32]. The $o b / o b$ genetic model offers the advantage of inducing 
obesity without using a HF diet, which can indirectly promote the absorption of lipophilic compounds—such as berberine and curcumin — thereby interfering with their potential interaction in the gut microbiota.

\section{Materials and Methods}

\subsection{Animals and Diet Intervention}

Thirty-six B6.V-Lep ob/ob JRj 6-week-old male mice (Janvier Labs, Saint Berthevin, France) were housed in groups of 3 per cage in specific pathogen-free and controlled environment (12-h daylight cycle) with free access to food and water. Mice were acclimated with a standard diet (AIN93M pellets, D10012Mi, Research Diet, New Brunswick, NJ, USA) for one week and then assigned into 4 groups of 9 mice: mice fed a standard diet (cc-/bb-, $n=9$ ), mice fed a standard diet supplemented with $0.1 \%$ berberine (cc-/bb+, $n=9)$, mice fed a standard diet supplemented with $0.3 \%$ curcumin $(\mathrm{cc}+/ \mathrm{bb}-, n=9)$ and mice fed $0.1 \%$ berberine and $0.3 \%$ curcumin $(\mathrm{cc}+/ \mathrm{bb}+, n=9)$. Eleonor (Waterloo, Belgium) supplied the berberine and curcumin extracts. The powdered form of the AIN-93M diet (D10012Mmi, Research Diet, New Brunswick, NJ, USA) was mixed with the compounds, following guidelines on Good Pharmacy Practice (method of successive addition, www.fip. org (accessed on 8 April 2021)). Sterile water was added in the different mixtures (300 mL water $/ \mathrm{kg}$ diet) to prepare pastes that were then divided into pellets $(2 \times 2 \times 0.5 \mathrm{~cm})$ and dried at room temperature. Food intake (for 3 mice per cage, $n=3$ ) and body weight were recorded twice a week. After 4 weeks of dietary treatment, 6-h-fasted mice were anaesthetized (isoflurane gas, Abbot, Ottignies, Belgium). Blood glucose was determined with a glucose meter (Roche Diagnostic, Meylan, France) on $3.5 \mu \mathrm{L}$ blood collected from the tip of the tail vein. Blood samples were harvested for further analysis. Mice were necropsied after cervical dislocation. Adipose tissues (epididymal, visceral, brown), liver, cecal content and tissue were weighted. Cecal content and tissue, colon and liver were frozen in liquid nitrogen and stored at $-80^{\circ} \mathrm{C}$. Animal experiments complied with the commonly accepted ' $3 \mathrm{Rs}^{\prime}$ ' and were approved and performed in accordance with the guidelines of the local ethics committee for animal care of the Health Sector of the Universite catholique de Louvain under the specific agreement number 2017/UCL/MD/005 (date of approval: 15/05/2017). Housing conditions were as specified by the Belgian Law of 29 May 2013, on the protection of laboratory animals (Agreement LA 1230314). All experiments were performed in strict accordance with relevant guidelines and regulations for the care and use of animals and in accordance with the EU directive.

\subsection{Gut Bacteria Analysis}

Genomic DNA was extracted from the cecal content using a QIAamp DNA Stool Mini Kit (Qiagen, Hilden, Germany) according to the manufacturer's instructions, including a bead-beating step. Quantification of total bacteria, Bacteroides spp., Bifidobacterium spp., Akkermansia spp. and Lactobacillus spp., was performed by qPCR, as described earlier [33].

\subsection{Biochemical Analysis}

Plasma triglycerides, cholesterol and non-esterified fatty acid (NEFA) concentrations were measured using kits coupling enzymatic reactions and spectrophotometric detections (SpectraMax i3x, Molecular Devices, UK) of end products (Diasys Diagnostic and Systems, Holzheim, Germany). Plasma insulin concentration was determined using ELISA kit (Mercodia, Upssala, Sweden). Triglycerides and cholesterol were measured in the liver tissue according to the Folch method [34] and using kits (Diasys Diagnostic and System, Holzheim, Germany). Alanine aminotransferase (ALAT) and aspartate aminotransferase (ASAT) levels were measured in the plasma as markers of liver damage using commercial kits according to the manufacturer's instructions (Diasys Diagnostic and Systems, Holzheim, Germany). 


\subsection{Tissue mRNA Analyses}

Total RNA was extracted from tissues using TriPure Isolation Reagent (Roche Diagnostics, Belgium). Complementary DNA was prepared by reverse transcription of $1 \mu \mathrm{g}$ of total RNA using the Reverse Transcription System (Promega, Madison, WI, USA). Real-time PCR was performed with a QuantStudio 3 Real-Time PCR System (Applied Biosystems, Den Ijssel, The Netherlands) using MasterMix GoTaq (Promega, WI, USA). Data were analyzed according to the $2-\Delta \Delta C T$ method. The purity of the amplified products was verified by analyzing the melting curve obtained at the end of the amplification step. The ribosomal protein L19 (Rp119) gene was used as a reference gene. Primer sequences are given in Supplementary Table S1.

\subsection{Statistical Analysis}

Results are presented as means with their standard errors. Statistical analysis was performed by two-way analysis of variance (ANOVA) followed by post-hoc Tukey's multiple comparison tests using Graphpad Prism software version 8 (San Diego, USA; www.graphpad.com (accessed on 8 April 2021)). The results were considered statistically significant when $p$ value was $<0.05$.

\section{Results}

3.1. Supplementation with Berberine Reduced Body Weight Gain and Food Intake of Ob/Ob Mice, Independently of Curcumin

Body weight of $o b / o b$ mice increased significantly with time, regardless of the dietary treatment (Supplementary Figure S1). Body weight gain of obese mice was lower upon berberine supplementation (Supplementary Table S1). This effect was linked to a lower food intake upon berberine intake, independently of curcumin intake (Table 1, Supplementary Figure S1). However, we did not find any significant effect of berberine on adiposity or liver weight. We observed a higher proportion of brown adipose tissue upon curcumin supplementation. The cecal content was significantly higher in the berberine groups. However, this effect was not accompanied by an increase in the cecal tissue.

Table 1. Body weight gain, food intake and organ weights.

\begin{tabular}{ccccc}
\hline & cc-/bb- & cc-/bb+ & cc+/bb- & cc+/bb+ \\
\hline Body weight gain (g) ${ }^{*}$ & $5.39 \pm 0.33^{\mathrm{a}}$ & $3.66 \pm 0.29^{\mathrm{b}}$ & $4.76 \pm 0.16^{\mathrm{ab}}$ & $3.64 \pm 0.40^{\mathrm{b}}$ \\
Total food intake (g/cage) & $93.9 \pm 2.6^{\mathrm{a}}$ & $82.4 \pm 2.2^{\mathrm{b}}$ & $94.1 \pm 3.2^{\mathrm{a}}$ & $79.9 \pm 0.6^{\mathrm{b}}$ \\
Visceral adipose tissue (g) & $1.11 \pm 0.05$ & $1.07 \pm 0.03$ & $1.17 \pm 0.04$ & $1.10 \pm 0.03$ \\
Epididymal adipose tissue (g) & $3.21 \pm 0.08$ & $3.24 \pm 0.14$ & $3.18 \pm 0.09$ & $3.05 \pm 0.06$ \\
Brown adipose tissue (g) \# & $0.21 \pm 0.01$ & $0.19 \pm 0.02$ & $0.24 \pm 0.01$ & $0.24 \pm 0.02$ \\
Liver (g) & $2.81 \pm 0.16$ & $2.89 \pm 0.13$ & $3.11 \pm 0.11$ & $2.78 \pm 0.10$ \\
Cecal content (g) * & $0.13 \pm 0.02$ & $0.16 \pm 0.01$ & $0.12 \pm 0.01$ & $0.14 \pm 0.01$ \\
Cecal tissue (g) & $0.050 \pm 0.002$ & $0.058 \pm 0.003$ & $0.054 \pm 0.003$ & $0.053 \pm 0.003$
\end{tabular}

$\mathrm{Ob} / \mathrm{ob}$ mice were fed a standard diet supplemented with or without berberine (bb) or curcumin (cc) for 4 weeks ( $n=9$ for each group). ${ }^{*} p<0.05$ for berberine effect and $\# p<0.05$ for curcumin effect (two-way ANOVA). Data with different superscript letters are significantly different at $p<0.05$ (Tukey post-hoc test).

\subsection{Supplementation with Berberine or Curcumin Has Prebiotic Potential in Ob/Ob Mice}

Berberine supplementation significantly decreased total bacteria in the cecal content, independently of the presence of curcumin in the diet (Figure 1a). The decrease in lactobacilli after berberine intake partially explained this effect (Figure 1b). Bacteroides spp. were not affected by the dietary treatments (Figure 1c). In contrast, Bifidobacterium spp. and Akkermansia spp. were increased by the berberine treatment (Figure 1d,e). Interestingly, the number of bifidobacteria was also significantly higher upon curcumin supplementation ( ${ }^{*} p<0.05$, two-way ANOVA). 

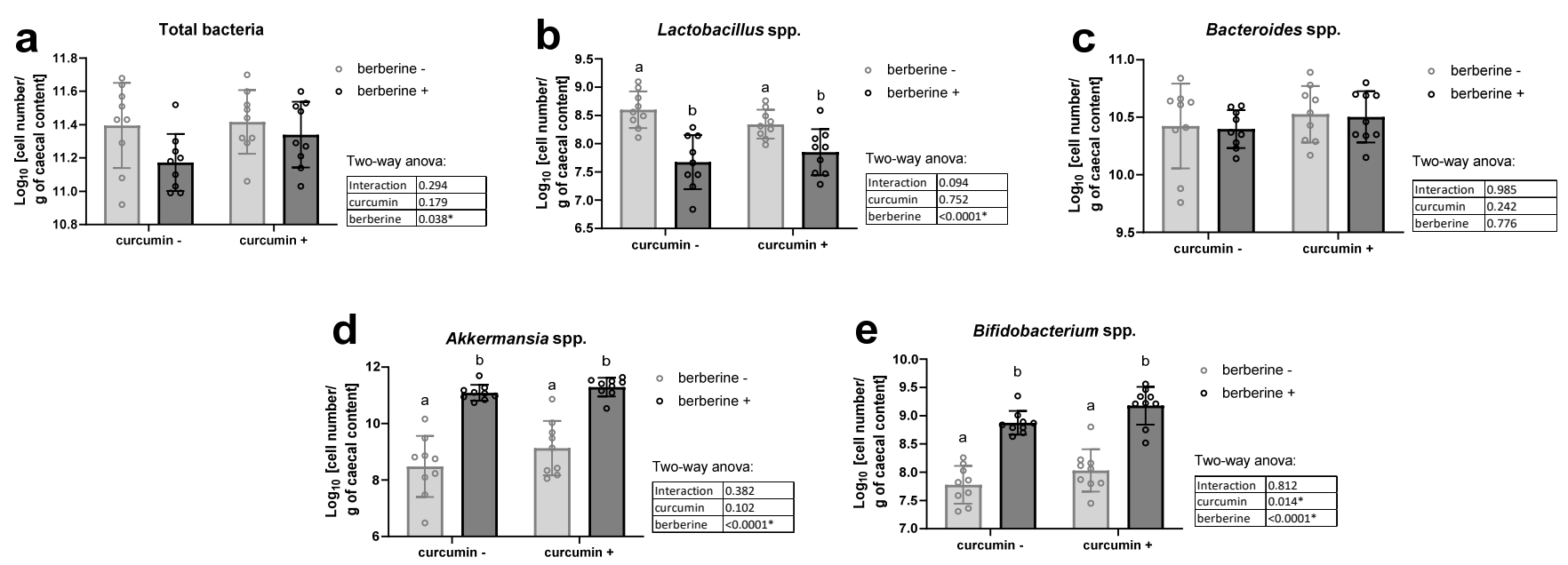

Figure 1. Bacteria in the cecal content. $O b / o b$ mice were fed a standard diet supplemented with or without berberine or curcumin for 4 weeks ( $n=9$ for each group). qPCR analysis of total bacteria (a), Lactobacillus spp. (b), Bacteroides spp. (c), Akkermansia spp. (d) and Bifidobacterium spp. (e) in the caecal content. ${ }^{*} p<0.05$ for berberine or curcumin effect (two-way ANOVA). Data with different superscript letters are significantly different at $p<0.05$ (Tukey post-hoc test).

\subsection{Supplementation with Berberine Upregulates the Expression of Host Peptides in the Colon of $\mathrm{Ob} / \mathrm{Ob}$ Mice}

We analyzed the mRNA levels of two colonic precursors of satietogenic peptides ( $G c g$ coding for glucagon-like peptides GLP-1 and GLP-2; Pyy coding for peptide YY), of four important inflammatory markers (Tnf, Il1b, Il6 and Ifng), as well as of peptides/proteins able to influence gut barrier function, such as $M u c 2$ involved in mucin production, and tight junction proteins (Tjp1 and Ocln) (Figure 2, Supplementary Table S2). Furthermore, we analyzed colonic expression of antimicrobial peptides, which can explain how nutrients modulate the gut ecosystem $[35,36]$. We measured the expression of antimicrobial peptides produced by enterocytes and Paneth cells in the colon: regenerating islet-derived 3-gamma (Reg3g), phospholipase A2g2 (Pla2g2a) and lysozyme C (Lyz) (Figure 2d, Supplementary Table S2). We observed decreased mRNA contents of Muc2, Pla2g2a and Gcg in the curcumin+/berberine- group. Interestingly, berberine increased the Pyy mRNA level and counteracted the decreased expression in genes induced by curcumin. In contrast, the expression of Reg $3 \gamma$ was downregulated upon berberine supplementation, mainly in the absence of curcumin in the diet, whereas the Lyz mRNA level was unchanged (Supplementary Table S2). Although occludin was also downregulated by curcumin supplementation, as observed above for other peptides, berberine intake did not influence its expression (Supplementary Table S2). The expression of a tight junction protein (Tjp1) and four important inflammatory markers in the colon of obese mice was not affected by dietary treatments (Supplementary Table S2).

3.4. Supplementation with Berberine Does Not Change Plasma Fasting Glucose and Insulin Levels but Decreases Triglyceridemia in $\mathrm{Ob} / \mathrm{Ob}$ Mice

Berberine alone or in combination with curcumin in the diet had no effect on fasting glycemia or fasting insulinemia in obese mice (Figure $3 a, b$ ). Of particular interest, berberine decreased plasma triglycerides of obese mice, independently of the presence of curcumin in the diet (Figure 3c). No effect was observed on cholesterolemia (Figure 3d). 
a

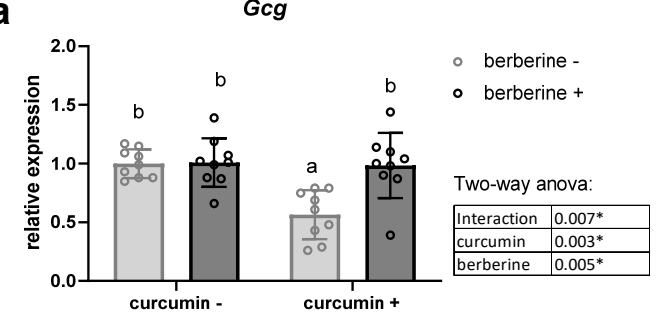

C

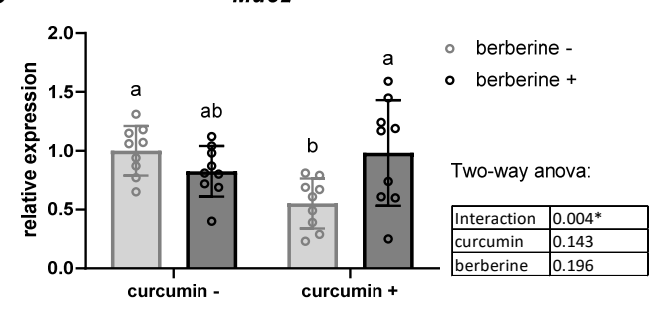

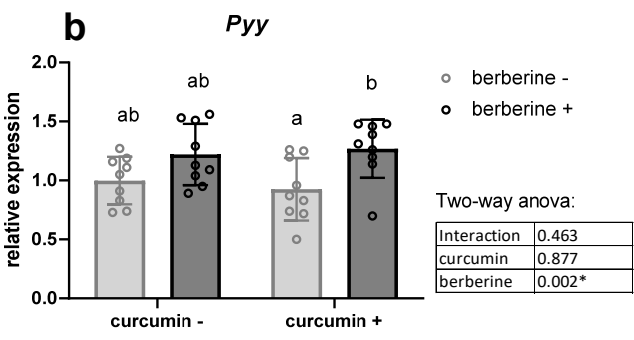

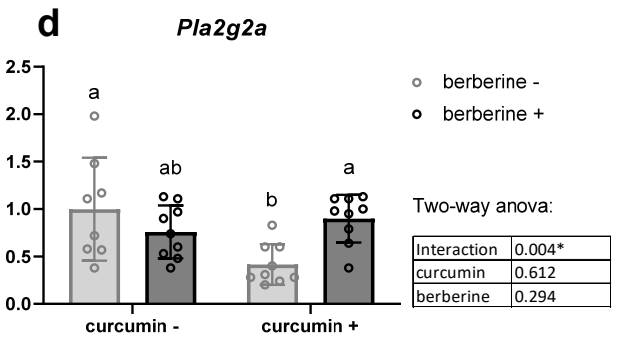

Figure 2. Colonic mRNA level coding for host peptides in the colon. Gcg coding for glucagon-like peptides (GLP-1, GLP-2) (a), Pyy coding for peptide YY (b), Muc2 coding for mucin 2 (c) and Pla2g2a coding for phospholipase A2 group II (d). Ob/ob mice were fed a standard diet supplemented with or without berberine or curcumin for 4 weeks $(n=9$ for each group). ${ }^{*} p<0.05$ for berberine or curcumin effect (two-way ANOVA). Data with different superscript letters are significantly different at $p<0.05$ (Tukey post-hoc test).

\section{a}

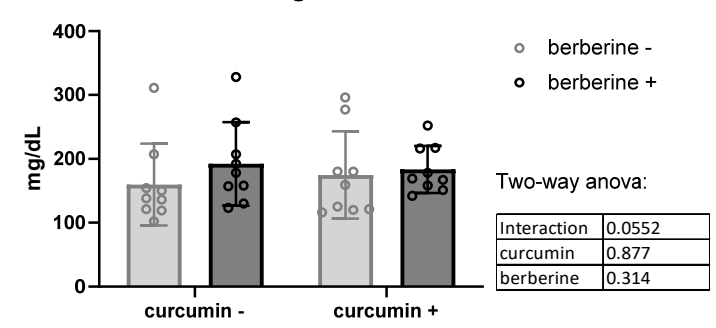

C

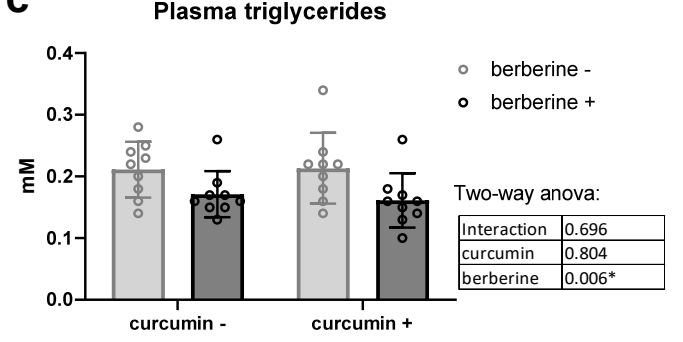

b
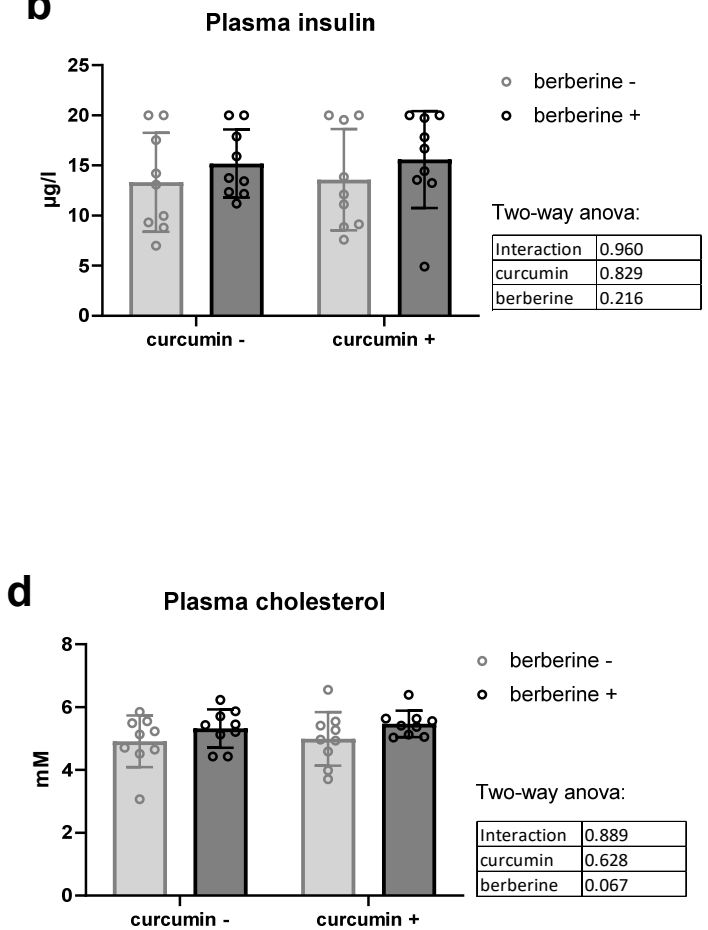

Figure 3. Lipid profile and glucose homeostasis of $o b / o b$ mice fed a standard diet supplemented with or without berberine or curcumin for 4 weeks ( $n=9$ for each group). Plasma glucose (a), plasma insulin (b), plasma triglycerides (c), plasma cholesterol $(\mathbf{d}) * p<0.05$ for berberine effect (two-way ANOVA). 


\subsection{Supplementation with Berberine Decreases Oxidative and Inflammatory Stresses in the Liver} of $\mathrm{Ob} / \mathrm{Ob}$ Mice

In accordance with the histological analysis (data not shown), the hepatic content of lipids (triglycerides and cholesterol) was not affected by the dietary treatments (Table 2). Although we observed the lowest triglyceride content in the liver of mice treated with curcumin only, this decrease was not significant and was even blunted by the presence of berberine in the diet. Importantly, berberine supplementation decreased the levels of ALAT, reflecting a lower hepatic injury in obese mice (Table 2). In line with this result, berberine treatment decreased markers of activated macrophages (Itgax coding for CD11c) and of oxidative stress (Nox1 coding for NADPH-oxidase). However, these results were not accompanied by lower expression of TNF $\alpha$, IL6, monocyte chemoattractant protein 1 (MCP1 or Ccl2) and toll-like receptor 4 (TLR4) (Table 2). Of note, the antioxidant effect of berberine was observed only in the presence of curcumin in the diet.

Table 2. Hepatic parameters related to lipid accumulation, inflammation and hepatotoxicity.

\begin{tabular}{|c|c|c|c|c|}
\hline & cc-/bb- & $\mathbf{c c}-/ \mathrm{bb}+$ & $\mathrm{cc}+/ \mathrm{bb}-$ & $\mathrm{cc}+/ \mathrm{bb}+$ \\
\hline $\operatorname{ALAT}(\mathrm{U} / \mathrm{L}) *$ & $150 \pm 12$ & $130 \pm 6$ & $158 \pm 11$ & $137 \pm 7$ \\
\hline Triglyceride content (nmol/mg tissue) $\S$ & $188 \pm 11$ & $171 \pm 10$ & $157 \pm 7$ & $182 \pm 6$ \\
\hline Cholesterol content (nmol/mg tissue) & $60 \pm 3$ & $55 \pm 3$ & $61 \pm 4$ & $60 \pm 2$ \\
\hline Tnf (relative expression) $\S$ & $1.00 \pm 0.08$ & $1.92 \pm 0.44$ & $2.36 \pm 0.61$ & $1.24 \pm 0.23$ \\
\hline Il6 (relative expression) $\S$ & $1.00 \pm 0.12$ & $1.70 \pm 0.21$ & $1.83 \pm 0.38$ & $1.27 \pm 0.19$ \\
\hline Ccl2 (relative expression) & $1.00 \pm 0.11$ & $0.98 \pm 0.12$ & $1.22 \pm 0.12$ & $1.11 \pm 0.27$ \\
\hline Tlr4 (relative expression) & $1.00 \pm 0.05$ & $1.35 \pm 0.26$ & $1.71 \pm 0.50$ & $1.69 \pm 0.65$ \\
\hline Itgax (relative expression) * & $1.00 \pm 0.14$ & $0.81 \pm 0.06$ & $1.19 \pm 0.16$ & $0.84 \pm 0.12$ \\
\hline Nox1 (relative expression) $* \S$ & $1.00 \pm 0.07^{\mathrm{a}}$ & $1.01 \pm 0.05^{\mathrm{a}}$ & $1.10 \pm 0.07^{\mathrm{a}}$ & $0.73 \pm 0.07^{b}$ \\
\hline
\end{tabular}

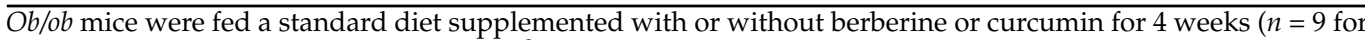
each group). ${ }^{*} p<0.05$ for berberine effect, $\S p<0.05$ for interaction effect (two-way ANOVA). Data with different superscript letters are significantly different at $p<0.05$ (Tukey post-hoc test). ALAT, alanine aminotransferase.

3.6. Increase in Beneficial Gut Bacteria upon Berberine or Curcumin Supplementation Correlates with Favorable Changes in Host Parameters in Ob/Ob Mice

We performed Spearman correlation analysis on data obtained from all mice $(n=36)$ in order to evaluate the potential links between the significant increase in specific gut bacteria induced by both phytochemicals (i.e., Bifidobacterium spp. and Akkermansia spp.) and all host parameters significantly affected (increased or decreased) mainly by the berberine treatment (Table 3). We observed significant negative correlations between body weight gain, triglyceridemia, parameters related to liver inflammation and oxidative stress with both Bifidobacterium spp. and Akkermansia spp. Moreover, colonic production of the satietogenic peptide YY was correlated with both beneficial Bifidobacterium spp. and Akkermansia spp.

Table 3. Correlation analysis between increased cecal bacteria and host parameters significantly modulated by berberin or curcumin treatments ${ }^{1}$.

\begin{tabular}{ccc}
\hline Spearman $\mathbf{r}$ & Bifidobacterium spp. & Akkermansia spp. \\
\hline Body weight gain & $-0.60^{*}$ & $-0.53^{*}$ \\
Plasma triglycerides & $-0.42^{*}$ & $-0.41^{*}$ \\
Brown adipose tissue weight & 0.05 & 0.10 \\
ALAT & -0.29 & -0.25 \\
Colonic Muc2 & 0.03 & 0.14 \\
Colonic Pla2g2a & 0.21 & 0.29 \\
Colonic Reg3g & -0.61 & $-0.58^{*}$ \\
Colonic Pyy & $0.39^{*}$ & $0.42^{*}$ \\
Colonic Gcg & 0.18 & 0.29 \\
Liver Nox1 & $-0.37^{*}$ & $-0.40^{*}$ \\
Liver Itgax & -0.32 & $-0.34^{*}$ \\
\hline
\end{tabular}

${ }_{1}^{1}$ Spearman $\mathrm{r}$ coefficients were computed between bacteria significantly increased after berberine and curcumin intake and all host parameters significantly affected by the dietary treatments $\left(n=36,{ }^{*} p<0.05\right)$. ALAT, alanine aminotransferase. 


\section{Discussion}

The gut microbiota is increasingly considered a symbiotic partner of health. Several data obtained in animal studies but also in humans suggest that the activity of the gut microbiota is a key factor to take into consideration when assessing the metabolic disorders related to obesity, such as insulin resistance, inflammation, dyslipidemia and MAFLD [1-3,37]. Emerging evidence has supported the relevance of some bacteria as hallmarks of obesity. The number of bifidobacteria has been inversely correlated with many disorders occurring upon obesity, such as adiposity, glucose intolerance and endotoxemia, notably in $o b / o b$ mice models [38,39]. Intervention studies with bifidobacteria as probiotics, or with prebiotics known to stimulate bifidobacteria growth, such as inulin and fructo-oligosaccharides, were tested as promising approaches to counteract some components of obesity and related pathologies [40]. Akkermansia muciniphila is attracting increasing interest for its health benefits [41]. In particular, Zhang et al. found a decreased abundance of Akkermansia muciniphila in diabetic and glucose-intolerant patients; this observation has been reported in several studies of obese individuals [42]. Among gut bacteria, Akkermansia is a mucus-colonizing bacterium involved in gut barrier function [43]. Treatment of rodents with $A$. muciniphila reduces obesity and related disorders, such as gut permeability, insulin resistance, glucose intolerance and steatosis. The hypothesis of specific changes in the abundance of bifidobacteria and Akkermansia in obesity is supported by several studies performed in mice as well as in humans $[1,6,44]$. To date, dietary fibers have been proposed as the key nutrients able to promote such interesting bacteria. In the present study, we extend the concept of prebiotics to non-carbohydrate phytochemicals. Previous studies using untargeted approaches to analyze the gut microbiota composition revealed that the main changes concerned with fostering the growth of Akkermansia spp., Bacteroides spp. and/or Lactobacillus spp. abundance in several animal models of metabolic disorders (HF diet-induced atherosclerosis in Apoe-/- mice, $d b / d b$ and KKAy diabetic mice) justified the targeting of those species when berberine impact on gut microbiota was investigated [45-47]. Therefore, we analyzed the gut microbiota composition using quantitative PCR targeting Akkermansia spp., Bifidobacterium spp., Lactobacillus spp. and Bacteroides spp., this methodology being more specific and quantitative than untargeted analyses (such as 16S rRNA gene sequencing). We have observed that the co-administration of curcumin and berberine exerted additive effects (i.e., no significant interaction in ANOVA as defined by Piggott et al., [48]) on Bifidobacterium spp. level. In addition, an important increase in Akkermansia was only observed after berberine intake, in line with other studies showing berberine's impact on this interesting bacterium [45-47,49]. The limitation is that we cannot exclude that other bacteria than the ones measured have been affected by the dietary treatments and may contribute to the effects demonstrated in the present study. For example, a detailed analysis of the gut microbiota in diabetic $d b / d b$ mice using $16 \mathrm{~s}$ rDNA high-throughput sequencing indicated that the proportions of Butyricimonas, Lactobacillus, Coprococcus and Ruminococcus increased in addition to Akkermansia, while the proportions of Prevotella and Proteus were reduced in the berberine-treated group [46]. Interestingly, in this last study, Bacteroides, Parabacteroides and Proteobacteria were positively correlated with weight or blood glucose, whereas Akkermansia, Sutterella, Clostridium, Butyricimonas and Bifidobacterium were negatively associated with these factors. Other bacteria belonging to Lachnospiraceae and Desulfovibrionaceae may also decrease upon berberine treatment in diabetic mice [45]. Furthermore, the abundance of Terrisporobacter and Helicobacter has been shown to increase while that of Pseudoflavonifractor, Mucisirillum, Alistipes, Ruminiclostridium and Lachnoclostridium decreased after berberine treatment in an animal model of alcoholic liver disease, even if $A$. muciniphila was the one that was most remarkably influenced by berberine administration [49]. By which mechanisms would berberine intake affect the composition of the gut microbiota? Multiple factors may contribute to changes in the intestinal microbiota, one of them being the interaction with the innate immune system of the gut. Antimicrobial molecules belonging to the innate immune system are secreted from epithelial cells. In the present study, the mRNA level of Pla2g2a in the colon 
increased, whereas that of Reg3g decreased upon berberine supplementation. Strikingly, although we found a decrease in Reg3g in this study, several previous studies have shown that direct supplementation with Akkermansia muciniphila increases the expression of Reg3g mRNA in the colon, and the higher abundance of Akkermansia observed upon prebiotic feeding was correlated with Reg3g [36,43,50,51]. This suggests that the host contributes to the modification of the gut microbiota induced by berberine extract supplementation, by modulating the production of such antimicrobial peptides [36,52]. However, whether Akkermansia directly influences the expression of Reg3g or vice versa remains to be investigated. In addition, we cannot exclude the direct antibacterial activity of curcumin and berberine since synergistic antimicrobial activity has already been demonstrated in vitro [31]; this mechanism can contribute, for example, to the lower proportion of Lactobacillus species observed in the cecal content mainly occurring in the curcumin+/berberin+ group. Altogether, these results suggest a prebiotic capacity of both chemicals in a genetic model of obesity.

Prebiotics such as fructans are able to prevent the overexpression of several host genes related to adiposity and inflammation, whereas they can increase the expression of colonic genes involved in the production of PYY, GLP-1 and GLP-2 in the portal vein, notably in $o b / o b$ mice in a 6-h-fasting state $[3,38,53]$. PYY and GLP-1 have a role in the control of appetite and glucose homeostasis, whereas GLP-2 contributes to the improvement of gut barrier function, namely through the higher expression of tight junction proteins. In the present study, we did not observe any increase in the tight junction proteins (TJP1, i.e., zonula occludens 1 ) or occludin mRNA levels under curcumin or berberine supplementation. However, we cannot exclude a modification by dietary treatments at the protein level, as already demonstrated with berberine supplementation by immunochemistry for occludin in the intestinal epithelium of obese rats due to HF feeding [54]. The gut barrier that controls the passage of gut-derived lipopolysaccharides (LPS) into the circulation is regulated by the intestinal inner mucus layer in addition to the presence of tight junctions. It was shown that $A$. muciniphila increases the mucus layer and its level is positively correlated with the number of mucin-producing goblet cells [43]. Here, we observed higher colonic expression of $M u c-2$ involved in mucin production in the curcumin+/berberine+ treated mice, suggesting an effect on the mucus layer, as already demonstrated for berberine, together with a higher abundance of Akkermansia spp. in HF diet-induced atherosclerosis Apoe-/- mice [47]. Importantly, we have shown higher colonic mRNA content of precursors of satietogenic peptides (Gcg and Pyy coding for GLP-1 and PYY, respectively) after berberine intake. These parameters have been measured after $6 \mathrm{~h}$ of fasting in accordance with previous protocols $[3,38,53]$. We do not know if similar effects would be relevant in the fed state; this could be one limitation of the outcome. The effect of berberine on preproglucagon and PYY expression was conditioned to the presence of curcumin in the diet. Of particular interest, berberine was reported to restore aberrant levels of gut hormones such as GLP-1, GLP-2 and PYY in the portal plasma of rats fed a HF diet [55]. The regulation of gut peptide production occurring in the colon of obese mice fed a diet enriched with berberine might be involved in the lower food intake observed in the present study, a mechanism that could participate in the lower body weight gain, as already described for inulin-type fructans $[3,53]$.

We have previously highlighted that plasma hypertriglyceridemia was dramatically reduced in $o b / o b$ mice treated with prebiotics for 5 weeks [39]. Here, this metabolic alteration was improved upon berberine supplementation after 4 weeks of treatment, independently of curcumin intake. However, 4 weeks of treatment with berberine or curcumin, at a concentration in the diet of $0.1 \%$ and $0.3 \%$, respectively, was insufficient to improve glucose homeostasis in obese mice in view of the unchanged levels of glycemia or insulinemia.

Concurrently with the increase in obesity, non-alcoholic fatty liver disease (NAFLD, recently defined as MAFLD) is becoming the most important cause of chronic liver disease, with a global prevalence of $24 \%$. However, no pharmacological therapy is currently available [56]. Diet plays an essential role in the development of MAFLD, but growing evidence 
suggests that the gut microbiota also has its part to play in the occurrence and evolution of this disease, notably through the production of specific bioactive metabolites $[57,58]$. The present work highlighted the hepatoprotective properties of supplementation with berberine. Berberine decreased oxidative (lower expression of Nox1 coding for NADPH-oxidase) and inflammatory events (lower expression of CD11c, a marker of activated macrophages) in the liver, leading to a lower hepatic injury (decrease in ALAT level in the plasma) in a genetic model of obesity and associated NAFLD, without improving steatosis and lipid homeostasis. It has also been reported that berberine supplementation may downregulate the levels of ALAT in rats fed a HF diet inducing obesity, indicating the restoration of liver function [54]. In this last study, and in line with our results, it was shown that berberine provided significant protection in NAFLD through ameliorating intestinal barrier function.

Finally, we highlighted significant correlations between Bifidobacterium and Akkermansia levels and the improvement of most metabolic alterations, including body weight gain, triglyceridemia, satietogenic peptide $Y Y$ expression, liver inflammation and oxidative stress.

In conclusion, these data supported the fact that non-carbohydrate phytochemicals may modulate the gut microbiota in obesity and related gut and hepatic alterations. This study thus strengthens the beneficial aspects of phytochemicals as prebiotic nutrients on body weight gain and food intake, an effect involving the production of satietogenic peptides in the colon. Moreover, these new prebiotic candidates improve obesity-related metabolic disorders such as hypertriglyceridemia, liver inflammation and hepatic oxidative injury when obesity is already established. It would be interesting to study the role of key metabolites produced by the gut microbiota from curcumin and berberine in order to propose the molecular mechanism(s) behind the systemic effects of these phytochemicals. Metabolomic approaches could be useful for this purpose. In addition, appropriate placebocontrolled intervention studies in overweight or obese individuals would also constitute a useful perspective to elaborate the proof of concept of their relevance in the management of obesity-related diseases in humans.

Supplementary Materials: The following are available online at https: / www.mdpi.com/article / 10.3390/nu13051436/s1. Figure S1. Body weight evolution and cumulative food intake; Table S1. Primers used in RT-qPCR; Table S2. Colonic mRNA levels coding for proteins or peptides involved in the regulation of innate immunity, gut barrier and/or inflammation.

Author Contributions: Conceptualization. A.M.N. and N.M.D.; methodology. A.M.N. and C.R.S.; software. A.M.N.; validation. J.R., P.D.C. and L.B.B.; formal analysis A.M.N. and C.R.S.; investigation. A.M.N. and C.R.S.; resources. P.D.C. and N.M.D.; data curation. A.M.N.; writing-original draft preparation. A.M.N.; writing-review and editing. A.M.N., J.R., P.D.C., L.B.B. and N.M.D.; visualization. A.M.N.; supervision. N.M.D.; project administration. A.M.N.; funding acquisition. N.M.D. All authors have read and agreed to the published version of the manuscript.

Funding: This research was financed by ELEONOR.

Institutional Review Board Statement: The study was conducted according to the guidelines of the local ethics committee for animal care of the Health Sector of the Université catholique de Louvain under the specific agreement number 2017/UCL/MD/005 (date of approval: 15/05/2017).

Informed Consent Statement: Not applicable.

Data Availability Statement: Data described in the manuscript will be made available upon reasonable request from the corresponding author.

Acknowledgments: We thank Bouazza Es Saadi and Isabelle Blave for their excellent technical assistance. Cándido Robles Sánchez (CRS) is a beneficiary of a postdoctoral fellowship program from Fundacion Alfonso Martin Escudero. NMD is a recipient of grants from the Service Public de Wallonie (SPW-EER, convention 1610365 and convention 8225), from the Fonds de la Recherche Scientifique (FRS-FNRS, convention PINT-MULTI R.8013.19 (NEURON, call 2019) and convention PDR T.0068.19) and from the Fédération Wallonie-Bruxelles (Action de Recherche Concertée ARC18-23/092). PDC is supported by the Fonds de la Recherche Scientifique (FNRS, FRFS-WELBIO: WELBIOCR-2019C-02R, FNRS T.0030.21 and EOS program no. 30770923). 
Conflicts of Interest: PDC is an inventor of patent applications dealing with the use of Akkermansia muciniphila and its components in the context of obesity and related disorders. PDC is co-founder of A-Mansia Biotech SA. The other authors declare no conflict of interest. The funders had no role in the design of the study, in the collection, analysis or interpretation of data, in the writing of the manuscript or in the decision to publish the results.

\section{References}

1. Delzenne, N.M.; Rodriguez, J.; Olivares, M.; Neyrinck, A.M. Microbiome response to diet: Focus on obesity and related diseases. Rev. Endocr. Metab. Disord. 2020, 21, 369-380. [CrossRef]

2. Delzenne, N.M.; Knudsen, C.; Beaumont, M.; Rodriguez, J.; Neyrinck, A.M.; Bindels, L.B. Contribution of the gut microbiota to the regulation of host metabolism and energy balance: A focus on the gut-liver axis. Proc. Nutr. Soc. 2019, 78, 319-328. [CrossRef]

3. Delzenne, N.M.; Cani, P.D. Interaction Between Obesity and the Gut Microbiota: Relevance in Nutrition. Annu. Rev. Nutr. 2011, 31, 15-31. [CrossRef]

4. Delzenne, N.M.; Neyrinck, A.M.; Cani, P.D. Modulation of the gut microbiota by nutrients with prebiotic properties: Consequences for host health in the context of obesity and metabolic syndrome. Microb. Cell Factories 2011, 10, S10. [CrossRef] [PubMed]

5. Druart, C.; Dewulf, E.M.; Cani, P.; Neyrinck, A.M.; Thissen, J.-P.; Delzenne, N.M. Gut Microbial Metabolites of Polyunsaturated Fatty Acids Correlate with Specific Fecal Bacteria and Serum Markers of Metabolic Syndrome in Obese Women. Lipids 2014, 49, 397-402. [CrossRef] [PubMed]

6. Depommier, C.; Everard, A.; Druart, C.; Plovier, H.; Van Hul, M.; Vieira-Silva, S.; Falony, G.; Raes, J.; Maiter, D.; Delzenne, N.M.; et al. Supplementation with Akkermansia muciniphila in overweight and obese human volunteers: A proof-of-concept exploratory study. Nat. Med. 2019, 25, 1096-1103. [CrossRef] [PubMed]

7. Plovier, H.; Everard, A.; Druart, C.; Depommier, C.; Van Hul, M.; Geurts, L.; Chilloux, J.; Ottman, N.; Duparc, T.; Lichtenstein, L.; et al. A purified membrane protein from Akkermansia muciniphila or the pasteurized bacterium improves metabolism in obese and diabetic mice. Nat. Med. 2017, 23, 107-113. [CrossRef]

8. Roberfroid, M.; Gibson, G.R.; Hoyles, L.; McCartney, A.L.; Rastall, R.; Rowland, I.; Wolvers, D.; Watzl, B.; Szajewska, H.; Stahl, B.; et al. Prebiotic effects: Metabolic and health benefits. Br. J. Nutr. 2010, 104, S1-S63. [CrossRef]

9. Delzenne, N.M.; Olivares, M.; Neyrinck, A.M.; Beaumont, M.; Kjølbæk, L.; Larsen, T.M.; Benítez-Páez, A.; Romaní-Pérez, M.; Garcia-Campayo, V.; Bosscher, D.; et al. Nutritional interest of dietary fiber and prebiotics in obesity: Lessons from the mynewgut consortium. Clin. Nutr. 2020, 39, 414-424. [CrossRef]

10. Vallianou, N.; Stratigou, T.; Christodoulatos, G.S.; Tsigalou, C.; Dalamaga, M. Probiotics, Prebiotics, Synbiotics, Postbiotics, and Obesity: Current Evidence, Controversies, and Perspectives. Curr. Obes. Rep. 2020, 9, 179-192. [CrossRef] [PubMed]

11. Gibson, G.R.; Roberfroid, M.B. Dietary Modulation of the Human Colonic Microbiota: Introducing the Concept of Prebiotics. J. Nutr. 1995, 125, 1401-1412. [CrossRef] [PubMed]

12. Gibson, G.R.; Hutkins, R.; Sanders, M.E.; Prescott, S.L.; Reimer, R.A.; Salminen, S.J.; Scott, K.; Stanton, C.; Swanson, K.S.; Cani, P.D.; et al. Expert consensus document: The International Scientific Association for Probiotics and Prebiotics (ISAPP) consensus statement on the definition and scope of prebiotics. Nat. Rev. Gastroenterol. Hepatol. 2017, 14, 491-502. [CrossRef]

13. Van Hul, M.; Cani, P.D. Targeting Carbohydrates and Polyphenols for a Healthy Microbiome and Healthy Weight. Curr. Nutr. Rep. 2019, 8, 307-316. [CrossRef] [PubMed]

14. Carrera-Quintanar, L.; Roa, R.I.L.; Quintero-Fabián, S.; Sánchez-Sánchez, M.A.; Vizmanos, B.; Ortuño-Sahagún, D. Phytochemicals that Influence Gut Microbiota as Prophylactics and for the Treatment of Obesity and Inflammatory Diseases. Mediat. Inflamm. 2018, 2018, 9734845. [CrossRef]

15. Singh, A.K.; Cabral, C.; Kumar, R.; Ganguly, R.; Rana, H.K.; Gupta, A.; Lauro, M.R.; Carbone, C.; Reis, F.; Pandey, A.K. Beneficial Effects of Dietary Polyphenols on Gut Microbiota and Strategies to Improve Delivery Efficiency. Nutrients 2019, 11, 2216. [CrossRef] [PubMed]

16. Álvarez-Martínez, F.J.; Barrajón-Catalán, E.; Encinar, J.A.; Rodríguez-Díaz, J.C.; Micol, V. Antimicrobial Capacity of Plant Polyphenols against Gram-Positive Bacteria: A Comprehensive Review. Curr. Med. Chem. 2020, 27, 2576-2606. [CrossRef] [PubMed]

17. Amiot, M.J.; Riva, C.; Vinet, A. Effects of dietary polyphenols on metabolic syndrome features in humans: A systematic review. Obes. Rev. 2016, 17, 573-586. [CrossRef]

18. Anhê, F.F.; Varin, T.V.; Le Barz, M.; Desjardins, Y.; Levy, E.; Roy, D.; Marette, A. Gut Microbiota Dysbiosis in Obesity-Linked Metabolic Diseases and Prebiotic Potential of Polyphenol-Rich Extracts. Curr. Obes. Rep. 2015, 4, 389-400. [CrossRef]

19. Sharma, R.; Gescher, A.; Steward, W. Curcumin: The story so far. Eur. J. Cancer 2005, 41, 1955-1968. [CrossRef] [PubMed]

20. Alappat, L.; Awad, A.B. Curcumin and obesity: Evidence and mechanisms. Nutr. Rev. 2010, 68, 729-738. [CrossRef] [PubMed]

21. Shehzad, A.; Ha, T.; Subhan, F.; Lee, Y.S. New mechanisms and the anti-inflammatory role of curcumin in obesity and obesityrelated metabolic diseases. Eur. J. Nutr. 2011, 50, 151-161. [CrossRef]

22. Nistico, S.; Tamburi, F.; Bennardo, L.; Dastoli, S.; Schipani, G.; Caro, G.; Fortuna, M.C.; Rossi, A. Treatment of telogen effluvium using a dietary supplement containing Boswellia serrata, Curcuma longa, and Vitis vinifera: Results of an observational study. Dermatol. Ther. 2019, 32, e12842. [CrossRef] 
23. Feng, D.; Zou, J.; Su, D.; Mai, H.; Zhang, S.; Li, P.; Zheng, X. Curcumin prevents high-fat diet-induced hepatic steatosis in apoe(-/-) mice by improving intestinal barrier function and reducing endotoxin and liver tlr4/nf-kappab inflammation. Nutr. Metab. (Lond.) 2019, 16, 79. [CrossRef]

24. Shao, W.; Yu, Z.; Chiang, Y.; Yang, Y.; Chai, T.; Foltz, W.; Lu, H.; Fantus, I.G.; Jin, T. Curcumin Prevents High Fat Diet Induced Insulin Resistance and Obesity via Attenuating Lipogenesis in Liver and Inflammatory Pathway in Adipocytes. PLoS ONE 2012, 7, e28784. [CrossRef] [PubMed]

25. Weisberg, S.P.; Leibel, R.; Tortoriello, D.V. Dietary Curcumin Significantly Improves Obesity-Associated Inflammation and Diabetes in Mouse Models of Diabesity. Endocrinology 2008, 149, 3549-3558. [CrossRef]

26. Kuo, J.-J.; Chang, H.-H.; Tsai, T.-H.; Lee, T.-Y. Positive effect of curcumin on inflammation and mitochondrial dysfunction in obese mice with liver steatosis. Int. J. Mol. Med. 2012, 30, 673-679. [CrossRef] [PubMed]

27. Zhong, Y.; Jin, J.; Liu, P.; Song, Y.; Zhang, H.; Sheng, L.; Zhou, H.; Jiang, B. Berberine Attenuates Hyperglycemia by Inhibiting the Hepatic Glucagon Pathway in Diabetic Mice. Oxidative Med. Cell. Longev. 2020, 2020, 6210526. [CrossRef]

28. Zhou, H.; Feng, L.; Xu, F.; Sun, Y.; Ma, Y.; Zhang, X.; Liu, H.; Xu, G.; Wu, X.; Shen, Y.; et al. Berberine inhibits palmitate-induced NLRP3 inflammasome activation by triggering autophagy in macrophages: A new mechanism linking berberine to insulin resistance improvement. Biomed. Pharmacother. 2017, 89, 864-874. [CrossRef]

29. Kim, W.S.; Lee, Y.S.; Cha, S.H.; Jeong, H.W.; Choe, S.S.; Lee, M.-R.; Oh, G.T.; Park, H.-S.; Lee, K.-U.; Lane, M.D.; et al. Berberine improves lipid dysregulation in obesity by controlling central and peripheral AMPK activity. Am. J. Physiol. Metab. 2009, 296, E812-E819. [CrossRef]

30. Li, H.; Qi, J.; Li, L. Phytochemicals as potential candidates to combat obesity via adipose non-shivering thermogenesis. Pharmacol. Res. 2019, 147, 104393. [CrossRef] [PubMed]

31. Bhatia, E.; Sharma, S.; Jadhav, K.; Banerjee, R. Combinatorial liposomes of berberine and curcumin inhibit biofilm formation and intracellular methicillin resistant Staphylococcus aureus infections and associated inflammation. J. Mater. Chem. B 2021, 9 , 864-875. [CrossRef]

32. Wexler, A.G.; Goodman, A.L. An insider's perspective: Bacteroides as a window into the microbiome. Nat. Microbiol. 2017, 2, 17026. [CrossRef]

33. Neyrinck, A.M.; Taminiau, B.; Walgrave, H.; Daube, G.; Cani, P.D.; Bindels, L.B.; Delzenne, N.M. Spirulina Protects against Hepatic Inflammation in Aging: An Effect Related to the Modulation of the Gut Microbiota? Nutrients 2017, 9, 633. [CrossRef] [PubMed]

34. Folch, J.; Lees, M.; Sloane-Stanley, G.H. A simple method for the isolation and purification of total lipides from animal tissues. J. Biol. Chem. 1957, 226, 497-509. [CrossRef]

35. Mukherjee, S.; Hooper, L.V. Antimicrobial Defense of the Intestine. Immunity 2015, 42, 28-39. [CrossRef] [PubMed]

36. Everard, A.; Lazarevic, V.; Gaïa, N.; Johansson, M.; Ståhlman, M.; Backhed, F.; Delzenne, N.M.; Schrenzel, J.; François, P.; Cani, P. Microbiome of prebiotic-treated mice reveals novel targets involved in host response during obesity. ISME J. 2014, 8, 2116-2130. [CrossRef]

37. Rodriguez, J.; Olivares, M.; Delzenne, N.M. Implication of the Gut Microbiota in Metabolic Inflammation Associated with Nutritional Disorders and Obesity. Mol. Nutr. Food Res. 2021, 65, e1900481. [CrossRef]

38. Cani, P.D.; Possemiers, S.; Van de, W.T.; Guiot, Y.; Everard, A.; Rottier, O.; Geurts, L.; Naslain, D.; Neyrinck, A.; Lambert, D.M.; et al. Changes in gut microbiota control inflammation in obese mice through a mechanism involving glp-2-driven improvement of gut permeability. Gut 2009, 58, 1091-1103. [CrossRef]

39. Everard, A.; Lazarevic, V.; Derrien, M.; Girard, M.; Muccioli, G.G.; Neyrinck, A.M.; Possemiers, S.; Van Holle, A.; François, P.; De Vos, W.M.; et al. Responses of Gut Microbiota and Glucose and Lipid Metabolism to Prebiotics in Genetic Obese and Diet-Induced Leptin-Resistant Mice. Diabetes 2011, 60, 2775-2786. [CrossRef]

40. Cerdó, T.; García-Santos, J.A.; Bermúdez, M.G.; Campoy, C. The Role of Probiotics and Prebiotics in the Prevention and Treatment of Obesity. Nutrients 2019, 11, 635. [CrossRef]

41. Cani, P.D.; de Vos, W.M. Next-generation beneficial microbes: The case of akkermansia muciniphila. Front. Microbiol. 2017, 8, 1765. [CrossRef] [PubMed]

42. Zhang, X.; Shen, D.; Fang, Z.; Jie, Z.; Qiu, X.; Zhang, C.; Chen, Y.; Ji, L. Human Gut Microbiota Changes Reveal the Progression of Glucose Intolerance. PLoS ONE 2013, 8, e71108. [CrossRef]

43. Everard, A.; Belzer, C.; Geurts, L.; Ouwerkerk, J.P.; Druart, C.; Bindels, L.B.; Guiot, Y.; Derrien, M.; Muccioli, G.G.; Delzenne, N.M.; et al. Cross-talk between Akkermansia muciniphila and intestinal epithelium controls diet-induced obesity. Proc. Natl. Acad. Sci. USA 2013, 110, 9066-9071. [CrossRef] [PubMed]

44. Delzenne, N.M.; Cani, P.; Everard, A.; Neyrinck, A.M.; Bindels, L.B. Gut microorganisms as promising targets for the management of type 2 diabetes. Diabetol. Clin. Exp. Diabetes Metab. 2015, 58, 2206-2217. [CrossRef] [PubMed]

45. Cao, H.; Li, C.; Lei, L.; Wang, X.; Liu, S.; Liu, Q.; Huan, Y.; Sun, S.; Shen, Z. Stachyose Improves the Effects of Berberine on Glucose Metabolism by Regulating Intestinal Microbiota and Short-Chain Fatty Acids in Spontaneous Type 2 Diabetic KKAy Mice. Front. Pharmacol. 2020, 11, 578943. [CrossRef]

46. Zhang, W.; Xu, J.-H.; Yu, T.; Chen, Q.-K. Effects of berberine and metformin on intestinal inflammation and gut microbiome composition in db/db mice. Biomed. Pharmacother. 2019, 118, 109131. [CrossRef] 
47. Zhu, L.; Zhang, D.; Zhu, H.; Zhu, J.; Weng, S.; Dong, L.; Liu, T.; Hu, Y.; Shen, X. Berberine treatment increases akkermansia in the gut and improves high-fat diet-induced atherosclerosis in apoe(-/-) mice. Atherosclerosis 2018, 268, 117-126. [CrossRef]

48. Piggott, J.J.; Townsend, C.R.; Matthaei, C.D. Reconceptualizing synergism and antagonism among multiple stressors. Ecol. Evol. 2015, 5, 1538-1547. [CrossRef]

49. Li, S.; Wang, N.; Tan, H.; Chueng, F.; Zhang, Z.; Yuen, M.; Feng, Y. Modulation of gut microbiota mediates berberine-induced expansion of immuno-suppressive cells to against alcoholic liver disease. Clin. Transl. Med. 2020, 10, e112. [CrossRef]

50. Régnier, M.; Rastelli, M.; Morissette, A.; Suriano, F.; Le Roy, T.; Pilon, G.; Delzenne, N.M.; Marette, A.; Van Hul, M.; Cani, P.D. Rhubarb Supplementation Prevents Diet-Induced Obesity and Diabetes in Association with Increased Akkermansia muciniphila in Mice. Nutrients 2020, 12, 2932. [CrossRef]

51. Hänninen, A.; Toivonen, R.; Pöysti, S.; Belzer, C.; Plovier, H.; Ouwerkerk, J.P.; Emani, R.; Cani, P.D.; De Vos, W.M. Akkermansia muciniphila induces gut microbiota remodelling and controls islet autoimmunity in NOD mice. Gut 2018, 67, 1445-1453. [CrossRef]

52. Gallo, R.L.; Hooper, L.V. Epithelial antimicrobial defence of the skin and intestine. Nat. Rev. Immunol. 2012, 12, 503-516. [CrossRef]

53. Delzenne, N.M.; Neyrinck, A.M.; Bäckhed, F.; Cani, P. Targeting gut microbiota in obesity: Effects of prebiotics and probiotics. Nat. Rev. Endocrinol. 2011, 7, 639-646. [CrossRef] [PubMed]

54. Li, D.; Zheng, J.; Hu, Y.; Hou, H.; Hao, S.; Liu, N.; Wang, Y. Amelioration of Intestinal Barrier Dysfunction by Berberine in the Treatment of Nonalcoholic Fatty Liver Disease in Rats. Pharmacogn. Mag. 2017, 13, 677-682. [CrossRef] [PubMed]

55. Xu, J.H.; Liu, X.Z.; Pan, W.; Zou, D.J. Berberine protects against diet-induced obesity through regulating metabolic endotoxemia and gut hormone levels. Mol. Med. Rep. 2017, 15, 2765-2787. [CrossRef] [PubMed]

56. Moon, A.M.; Singal, A.G.; Tapper, E.B. Contemporary Epidemiology of Chronic Liver Disease and Cirrhosis. Clin. Gastroenterol. Hepatol. 2020, 18, 2650-2666. [CrossRef] [PubMed]

57. Knudsen, C.; Neyrinck, A.M.; Lanthier, N.; Delzenne, N.M. Microbiota and nonalcoholic fatty liver disease: Promising prospects for clinical interventions? Curr. Opin. Clin. Nutr. Metab. Care 2019, 22, 393-400. [CrossRef] [PubMed]

58. Canfora, E.E.; Meex, R.C.R.; Venema, K.; Blaak, E.E. Gut microbial metabolites in obesity, NAFLD and T2DM. Nat. Rev. Endocrinol. 2019, 15, 261-273. [CrossRef] 